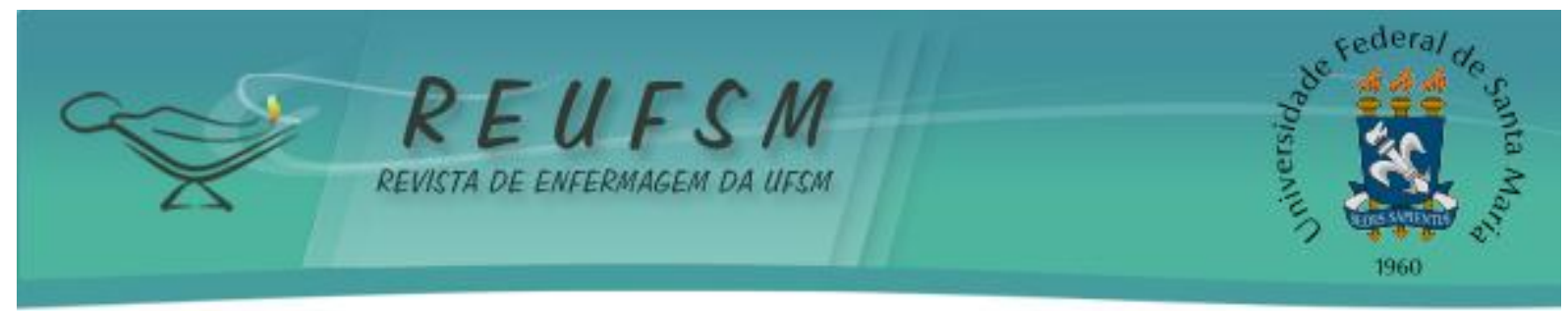

ARTIGO ORIGINAL

\title{
O ESTADO NUTRICIONAL E COMPORTAMENTO ALIMENTAR DE UM GRUPO DE MULHERES NA ESTRATÉGIA DE SAÚDE DA FAMÍLIA
}

FEEDING BEHAVIOR OF A GROUP OF WOMEN IN FAMILY HEALTH STRATEGY

\section{EL ESTADO NUTRICIONAL Y COMPORTAMIENTO ALIMENTAR DE UN GRUPO DE MUJERES EN LA ESTRATEGIA DE SALUD DE LA FAMILIA}

Viviane Euzébia Pereira Santos ${ }^{1}$

Nayara Mendes $\mathrm{Cruz}^{2}$

Laísla Alves Moura ${ }^{3}$

Jaqueline Gonçalves Moura ${ }^{4}$

Khésia Kelly Cardoso Matos ${ }^{5}$

RESUMO: Objetivo: analisar o estado nutricional e o comportamento alimentar de mulheres em idade adulta de unidades de Estratégia de Saúde da Família. O comportamento alimentar é um dos fatores condicionantes do estado nutricional e, consequentemente, da saúde física e psicológica. Método: pesquisa quantitativa, desenvolvida a partir de um estudo de caso coletivo, em que a coleta de dados ocorreu mediante entrevistas semiestruturadas com questões abertas e fechadas, no período de janeiro e fevereiro de 2008. A população estudada foi composta por 58 mulheres. Resultados: verificou-se que a média de idade das entrevistadas é de 42 anos, $91,4 \%$ tem filhos, $56,9 \%$ não praticam atividades físicas. Os resultados demonstram, ainda, que $51,7 \%$ das mulheres estão com o peso normal, $19 \%$ com sobrepeso e 29,3\% com obesidade. Conclusão: comprovando, dessa forma que a obesidade, cada vez mais, torna-se um dos principais problemas da saúde pública.

Descritores: Comportamento alimentar; Estado nutricional; Mulheres.

ABSTRACT: Objective: to analyze the nutritional state and feeding behavior in adult women in units of Family Health Strategy. Since eating is one of the factors conditioning the nutritional status and, consequently, the physical and psychological health. Method: quantitative research developed from a collective case study, in which the data were collected through semi-structured interviews with open and closed questions between January and February 2008. The study population comprised 58 women and data collection was conducted through semi-structured interview, during January and February 2008. Results: from the interview found that the average age of the interviews is 42 years, $91.4 \%$ have children, 56,9\% did not practice physical activities. The results show also that $51.7 \%$ of women with normal weight, with about 19 weight\% and $29.3 \%$ were obese. Conclusion: proving thus that obesity increasingly becomes a major public health problem.

Descriptors: Feeding behavior; Nutritional status; Women.

\footnotetext{
${ }^{1}$ Doutora em Enfermagem. Professora Permanente do Departamento de Enfermagem e Pós Graduação em Enfermagem e Vice-Líder do grupo de pesquisa laboratório de investigação do cuidado, segurança e tecnologias em saúde e enfermagem da Universidade Federal do Rio Grande do Norte. E-mail: vivianeepsantos@gmail.com ${ }^{2}$ Estudante de graduação em enfermagem. Universidade Federal do Vale do São Francisco (UNIVASF). E-mail: nayaramendescruz@hotmail.com

${ }^{3}$ Estudante de graduação em enfermagem. Universidade Federal do Vale do São Francisco (UNIVASF). E-mail: laislaalves19@gmail.com

${ }^{4}$ Estudante de graduação em enfermagem. Universidade Federal do Vale do São Francisco (UNIVASF). E-mail: enf.jaquelinemoura@gmail.com

${ }^{5}$ Estudante de graduação em enfermagem. Universidade Federal do Vale do São Francisco (UNIVASF). E-mail: keumatos3@hotmail.com
} 


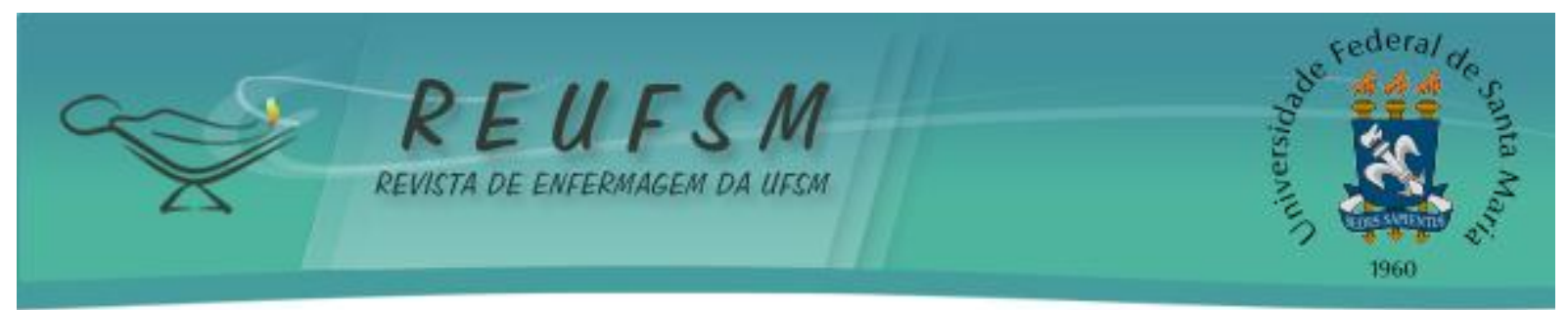

RESUMEN: Objetivo: este artículo trata de un análisis descriptivo para evaluar el estado nutricional y el comportamiento de alimentación de las mujeres adultas en las unidades de la Estrategia de Salud Familiar. El comportamiento alimentar es uno de los factores que condicionan el estado nutricional y, en consecuencia, la salud física y psicológica. Método: la investigación cuantitativa desarrollada a partir de un estudio de caso colectivo, y los datos fueron recogidos a través de entrevistas semiestructuradas con preguntas abiertas y cerradas entre enero y febrero de 2008. La población de estudio incluyó 58 mujeres. Resultados: desde la entrevista encontró que la edad media de las entrevistas es de 42 años, el 91,4\% tienen hijos, el 56,9\% no practican actividades físicas. Los resultados muestran también que el $51,7 \%$ de las mujeres con peso normal, el $19 \%$ con sobrepeso y $29,3 \%$ son obesas. Conclusión: demostrando así que la obesidad, cada vez se convierten en un problema de salud pública.

Descriptores: Conducta alimentaria; Estado nutricional; Mujeres.

\section{INTRODUÇÃO}

A alimentação constitui-se em uma das atividades mais importantes para o homem. Sua ligação aos aspectos da saúde dos indivíduos vem sendo estudada há anos pela ciência, a qual a cada dia encontra maiores evidências e inter-relações para essas questões. Recentemente, estudos indicaram que a alimentação influencia diretamente a saúde das pessoas, tanto positiva como negativamente, e é apontada como um dos fatores mais importantes para a longevidade com qualidade de vida.

Em relação à realidade alimentar brasileira, esta é marcada por contrastes, apresentando, simultaneamente, problemas causados tanto pela falta quanto pelo excesso de alimentos. Estabelece-se, desta forma, um antagonismo de tendências temporais entre desnutrição e obesidade, definindo uma das características marcantes do processo de transição nutricional no país.

O comportamento alimentar do ser humano é um dos fatores condicionantes mais próximos do seu estado nutricional. Corresponde não apenas aos chamados "hábitos alimentares", como também a todas as práticas relativas à alimentação desse indivíduo, como seleção, aquisição, conservação, preparação e consumo efetivo de alimentos.

A conduta alimentar, normalmente, tem suas bases fixadas na infância e adolescência, transmitidas pela família, sustentadas pela tradição, crenças, valores e tabus, que passam através das gerações.

As condições de peso da população, segundo pesquisas realizadas pela Organização Mundial de Saúde, revelam que o sobrepeso e a obesidade, vêm tornando-se uma epidemia com alta incidência e prognóstico ruim para a saúde pública. De acordo com os dados fornecidos, cerca de 15 milhões de crianças e populações infanto-juvenis estão acima do peso ideal, representando que $15 \%$ desta população encontra-se com excessivo peso.

Deste problema decorrem várias considerações importantes, dentre as quais, pode-se citar a existência de uma série de agravos à saúde, uma vez que a obesidade, a inatividade física, a hipertensão arterial e o tabagismo são os principais fatores de risco para as doenças cardiovasculares.

Um relato sobre dieta alimentar, nutrição e preservação de doenças crônicas, da Organização Mundial da Saúde, com a Organização para a Alimentação e Agricultura das Nações Unidas (FAO), aponta que as doenças associadas à obesidade contribuíram com aproximadamente $46 \%$ das doenças e $59 \%$ das mortes por esses fatores a nível mundial. ${ }^{6}$

Com isso, este estudo teve como questão norteadora: qual o estado nutricional e o 


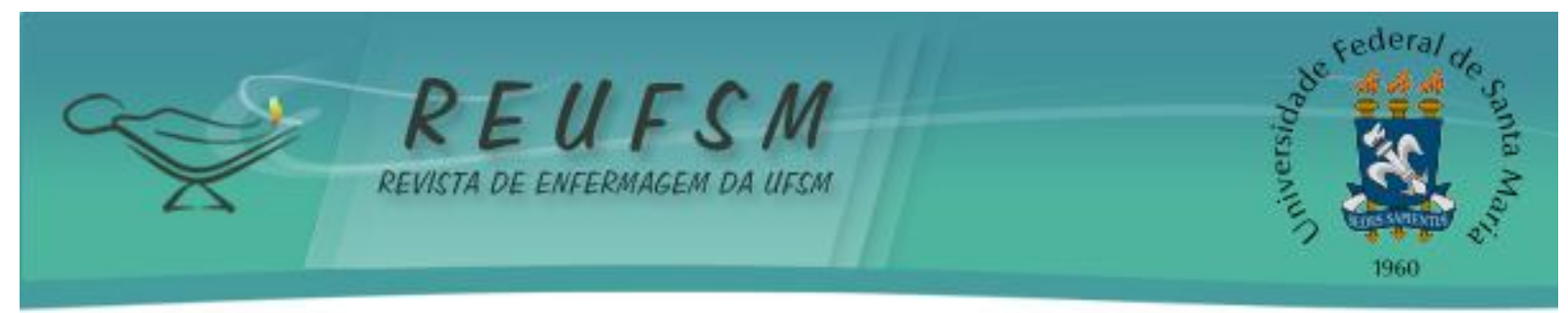

comportamento alimentar das mulheres que frequentam as Unidades de Estratégia de Saúde da Família de Joinville? E como objetivo analisar o estado nutricional e o comportamento alimentar de mulheres em idade adulta que frequentam Unidades de Estratégia de Saúde da Família.

0 interesse em pesquisar o comportamento alimentar das mulheres deve-se ao difícil cotidiano de tantas mulheres que são marcados pela dupla jornada de trabalho, incluindo os afazeres domésticos, os cuidados com os filhos, as ocupações do trabalho. Dessa forma, há necessidade de investigar a relação entre o consumo alimentar e obesidade ou sobrepeso, especialmente, com as mudanças ocorridas na dieta da população brasileira, a chamada dieta "moderna". ${ }^{8}$

\section{MÉTODO} caso coletivo.

Trata-se de uma pesquisa quantitativa, desenvolvida por meio de um estudo de

0 estudo quantitativo é aquele que tende a classificar, analisar e traduzir, em número, opiniões, reações, hábitos e atitudes de um grupo pré-estabelecido, necessitando, para isso, de recursos e técnicas estatísticas. ${ }^{9}$

A população deste estudo foi constituída por 58 mulheres de duas Unidades de Estratégia de Saúde da Família de Joinville, Santa Catarina. O critério para participar do estudo era ser a primeira mulher em idade adulta (19 a 59 anos 11 meses e 29 dias) que fosse atendida no período de 22/01/2008 a 29/02/2008.

Seguindo a resolução 196/96, do Conselho Nacional Saúde (CNS), este estudo só ocorreu após aprovação pelo comitê de ética da instituição, Protocolo $n^{\circ} 32 / 2007$, e do preenchimento do termo de consentimento livre e esclarecido, que garante o direito de participar ou desistir a qualquer momento da pesquisa, mantendo o anonimato das participantes.

A coleta de dados foi realizada por meio de entrevista semiestruturada com questões abertas e fechadas, contendo itens de identificação, dados socioeconômicos, preferência e rejeições alimentares, e do diagnóstico nutricional determinado pelo peso e estatura, para cálculo do IMC (Índice da Massa Corporal).

Os dados foram analisados, quantitativamente, mediante estatística descritiva (frequências absoluta e relativa), tabulados e analisados em planilhas descritivas do Microsoft Excel 2007.

\section{ANÁLISE E DISCUSSÃO DOS DADOS}

A população estudada constituiu-se de 58 mulheres em idade adulta que frequentaram as duas Unidades de Estratégia de Saúde da Família. A média de idade das mulheres foi de 42 anos, sendo a maioria vinda de outras regiões do estado, Santa Catarina, e estados vizinhos. Verificou-se que $84,48 \%$ da população estudada residiam com marido e $91,4 \%$ responderam ter filhos.

Os hábitos ou "estilo de vida", expressos por práticas cotidianas, não são iguais para todos, mas também não são puramente atitudes individuais conscientes, isoladas e imutáveis. ${ }^{11}$ Portanto, a companhia de outras pessoas, na moradia, está diretamente relacionada ao consumo alimentar e estilo de vida.

Observa-se que a ocorrência de obesidade entre membros de uma mesma família é conhecida, acreditando-se que isso possa ser relacionado tanto a fatores genéticos quanto aos hábitos de vida. A tendência de obesidade dos pais leva a um risco aumentado de obesidade nos filhos. 


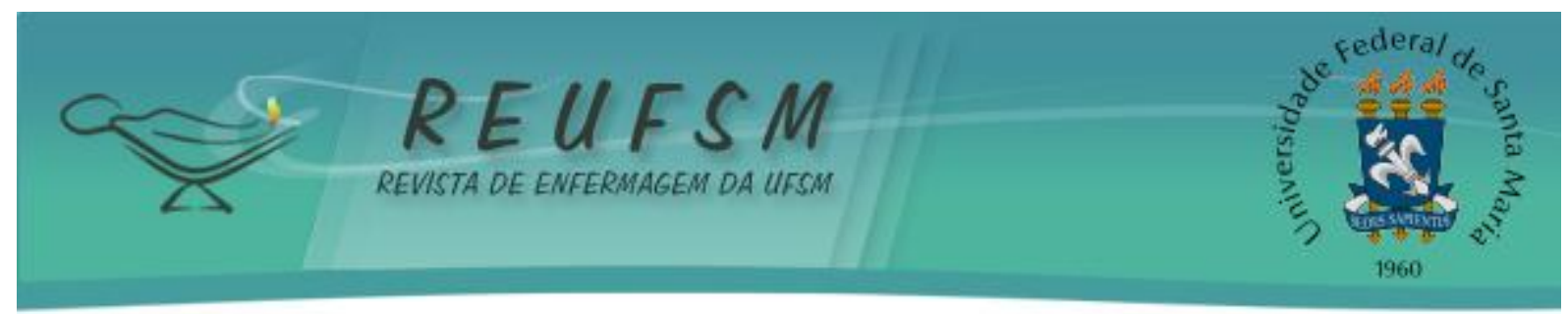

Aquilo que se come e se bebe não é só uma questão de escolha individual. A pobreza, a exclusão social e a qualidade da informação disponível frustram ou, pelo menos, restringem a escolha de uma alimentação mais adequada e saudável. E o que se come e se bebe é ainda, em grande parte, uma questão familiar e social. Em geral, contrariamente, ao que se pode imaginar, as escolhas alimentares são determinadas não tanto pela preferência e pelos hábitos, mas pelo sistema de produção e de abastecimento de alimentos.

Neste estudo, observou-se que 51,7\% das mulheres estão com o peso normal (IMC de 19 a 25), $19 \%$ com sobrepeso e $29,3 \%$ das mulheres em idade adulta estão obesas (IMC acima de 30).

Os dados mostraram que a atividade física não é um preceito para a maioria das mulheres. Ao responderem o questionário, $56,9 \%$ dessas não praticavam atividades físicas, e das que praticavam $32 \%$ faziam até duas vezes por semana.

A prática regular de atividades físicas está associada à diminuição de morbidades tais como obesidade, hipertensão arterial, diabetes. Estudos mostraram fortes relações entre a menor frequência de atividade física e presença de doenças crônicas não transmissíveis. ${ }^{1416}$

Ao questionar as mulheres sobre seu comportamento alimentar, parte delas referiu omitir alguma refeição, tendo, a maioria, uma média de 3,68 refeições diárias. Já em um estudo realizado, no Rio de Janeiro, sobre a propaganda de alimentos, o qual analisou o consumo alimentar e gasto energético em mulheres adultas, avaliou que as mulheres entrevistadas mencionaram uma média de quatro refeições diárias. ${ }^{17}$

0 papel feminino na sociedade atual ainda é relacionado aos cuidados domésticos, ou seja, entre outras atividades a mulher tem a responsabilidade no preparo do alimento para a família. Nas respostas encontradas, $82,7 \%$ das mulheres preparam os alimentos em suas casas. Em estudo realizado com mulheres donas-de-casa e trabalhadoras verificou-se que a atividade doméstica mais assumida pelas mulheres, é de cozinhar (donas-de-casa: 72,3\%; trabalhadoras: $37,7 \%$ ).

Ao investigar sobre gostar ou não de provar alimentos novos, $72,4 \%$ das mulheres responderam positivamente e $27,6 \%$ referiram preferir os alimentos que já conheciam. Porém, ao perguntar se havia influência da mídia na compra de alimentos, observou-se $87,9 \%$ de respostas negativas e apenas $12,1 \%$ de positivas.

Acredita-se que os meios de comunicação exerce papel fundamental na formação de novos hábitos alimentares ${ }^{19}$, porém as entrevistadas negam sofrer influência da mídia.

Além dos meios de comunicação, existem vários fatores que podem influenciar na escolha dos alimentos, como: a vontade no momento, a qualidade, a aparência, as características nutricionais, o preço e os ingredientes ou todas as opções. No presente estudo $44,8 \%$ responderam que observam todos os itens na escolha de um alimento. Outras $17,2 \%$ apontaram o preço como a prioridade de escolha. Só $8,6 \%$ declararam observar as características nutricionais dos alimentos.

Para uma alimentação saudável é de suma importância a consulta e a interpretação da informação nutricional e da lista de ingredientes presentes nos rótulos dos alimentos.

0 estado nutricional dos indivíduos pode ser influenciado pelas técnicas utilizadas no preparo dos alimentos. Entre as respostas dos questionamentos, sobre o comportamento alimentar das mulheres, a técnica de preparo preferida foi assado/grelhado $(44,8 \%)$ e a técnica mais utilizada foi o cozido $(36,2 \%)$.

Os resultados encontrados supostamente estão relacionados com o tempo de preparo de cada técnica, pois a vida agitada das mulheres no cuidado da casa e das que trabalham fora, muitas vezes, não permite que sua técnica preferida seja utilizada. As porcentagens de preferência e utilização da fritura entre as mulheres foram de $18,9 \%$ e $24,1 \%$, considerada alta, já que são elas que preparam o alimento na maioria das vezes em suas casas. Há impactos negativos de dietas com alto teor calórico, pois as pessoas podem 


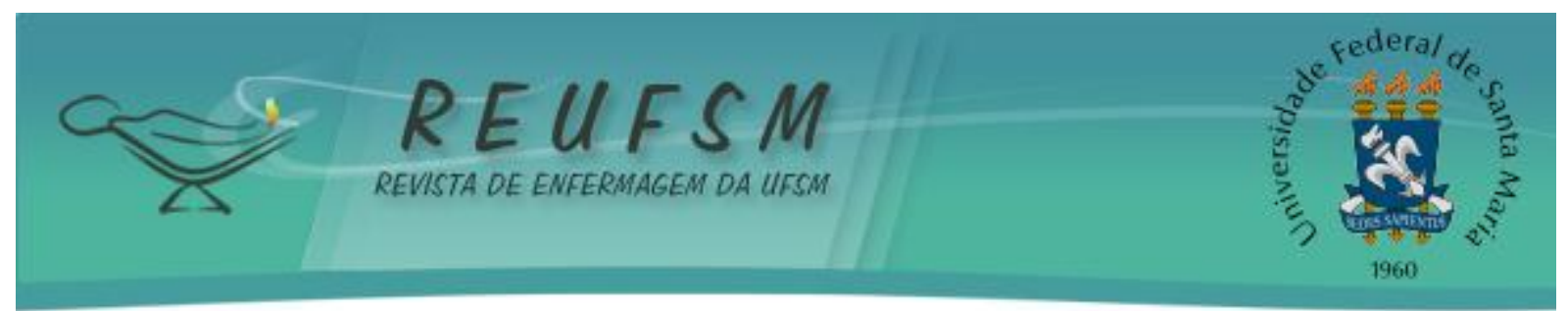

apresentar alto risco para desenvolver doenças cardiovasculares. ${ }^{14}$

No que se refere a ter ou não preconceito em relação a algum tipo de alimento, 63,8\% responderam positivamente. Dentre eles, $40 \%$ responderam ter preconceito com frutos do mar. Este resultado não era esperado por se tratar de um estudo com mulheres de uma cidade litorânea. Porém observou-se que a procedência das mulheres é bastante diversificada, sendo que os frutos do mar podem não estar entre os hábitos alimentares dos seus locais de origem.

Nesta pesquisa $39,7 \%$ das mulheres responderam que os alimentos tradicionais no consumo de sua família são feijão e arroz, alimentos de alto valor nutricional. ${ }^{20} \mathrm{Um}$ número pouco significante, pois considerando a importância nutricional da combinação feijão e arroz, ela deve ser resgatada ou mantida, valorizada e incentivada como elemento central da alimentação da população brasileira.

Em relação à situação socioeconômica das mulheres entrevistadas, 48,3\% tinham uma renda familiar mensal de até três salários mínimos. Considerando que a renda influencia diretamente ao tipo de alimentação, já que a mesma vai depender do poder aquisitivo dessas mulheres.

Fatores sociais, destacando o novo papel feminino e sua inserção no mercado de trabalho, são comprovados nesta pesquisa, na qual $60,4 \%$ das mulheres responderam que têm atividade laboral formal. De acordo com a OMS, o número de mulheres que conciliam trabalho fora de casa e os cuidados da família tem aumentado, na mesma proporção em que declinam a motivação, tempo e energia para exercer todas essas atividades. ${ }^{21}$

Nas entrevistas, 39,6\% das mulheres mencionaram ter frequentado a escola por até quatro anos, e 19,3\% por até oito anos. Inquéritos demonstraram que a relação entre o número de anos de estudo é inversa à prevalência de obesidade, ou seja, a prevalência de obesidade diminui com o aumento do número de anos cursados. ${ }^{22-24}$

Alterações nas condições sociais e demográficas, abrangendo o público feminino, vêm provocando diversas alterações nos hábitos alimentares deste grupo. A inserção da mulher no mercado de trabalho, a concentração das populações no meio urbano e a diminuição de esforço físico e a crescente industrialização dos alimentos, condicionam mudanças na alimentação e está diretamente relacionada à maior prevalência da obesidade.

\section{CONSIDERAÇÕES FINAIS}

Constatou-se que, as mulheres em seu papel na sociedade atual, ainda têm o grande poder de interferir nos hábitos de sua família. Um desequilíbrio na alimentação e no estilo de vida dessas pode repercutir em seu estado nutricional e de saúde e, consequentemente, predispor às famílias a doenças crônicas não transmissíveis.

0 presente estudo chama a atenção para a incidência de sobrepeso e obesidade das mulheres. Contudo, a detecção precoce dos fatores de risco para doenças crônicas não transmissíveis é primordial para atuação como estratégia de promoção e prevenção da saúde.

0 perfil nutricional e de saúde, das mulheres em idade adulta, nas duas unidades, demonstrou a necessidade de programas educacionais de intervenção para adoção de um estilo de vida mais saudável, promovendo assim uma melhoria na qualidade de vida.

A Estratégia de Saúde da Família (ESF) tem como objetivo geral melhorar o estado de saúde da população, mediante a construção de um modelo assistencial de atenção, baseado na promoção e proteção, no diagnóstico precoce, na recuperação da saúde dos indivíduos e da família.

Portanto, é necessário que o município em questão possua uma equipe multiprofissional para referência, contendo também profissionais como nutricionista e educador físico, os quais poderiam estar contribuindo com as equipes da ESF, ao oportunizarem de forma conjunta, uma assistência integral para as famílias. 


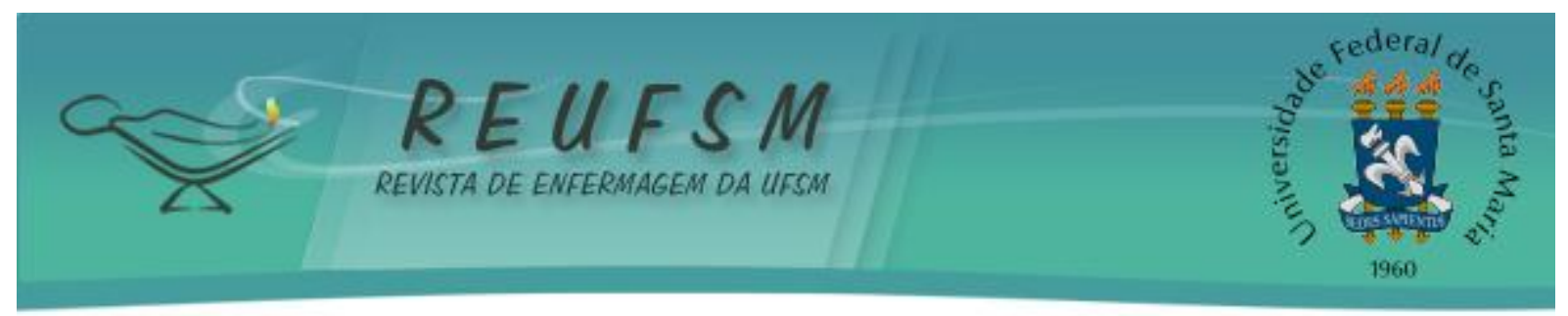

Além disso, sugere-se para a Secretaria de Saúde do município a implantação de um sistema de vigilância alimentar e nutricional para as mulheres em todas as faixas etárias. Visto que, as maiores frequentadoras das unidades de saúdes são as mulheres e que apesar destas estarem, cada vez mais, inseridas no mercado de trabalho, ainda são as responsáveis por prover e preparar a alimentação da família, com isso os comportamentos alimentares seguidos acabam interferindo na saúde de toda família.

\section{REFERÊNCIAS}

1. Guimarães A, Paranaguá MMM, Miyahira N, Pinheiro F, Castro M. Tendências do food service: oferecer alimentação saudável. Nutrição em Pauta. 2001;10(47):8-13.

2. Costa PRF, Assis AMO, Silva MCM, Santana MLP, Dias JC, Pinheiro SMC, et al. Mudança nos parâmetros antropométricos: a influência de um programa de intervenção nutricional e exercício físico em mulheres adultas. Cad Saúde Pública, Rio de Janeiro. 2009;25(8):1763-73.

3. Silva JG, Teixeira MLO, Ferreira MA. Alimentação e saúde: sentidos atribuídos por adolescentes. Esc Anna Nery Rev Enferm. 2012;16(1):88-95.

4. Batista Filho M, Rissin A. A transição nutricional no Brasil: tendências regionais e temporais. Cad. Saúde Pública. 2003;19(Supl 1):S181-S91.

5. Motta DG. Educação nutricional. São Paulo: IBRASA; 1987.

6. Organização Mundial de Saúde (OMS). Comunicado de Imprensa Conjunto da OMS/FAO [internet]. Geneva: Organização Mundial da Saúde. [acesso em 2005 ago10]. Disponível em: < http://who.int/nutrition/publications/pressrelease32_pt.pdf >.

7. Krauzer IM, Brocardo D, Scarsi T. A metodologia de Callista Rroy aplicada em clientes submetidos à intervenção hemodinâmica. Rev Enferm UFSM [internet]. 2011 [acesso em mar 11];1(2):183-193. Disponível em: http://cascavel.ufsm.br/revistas/ojs2.2.2/index.php/reufsm/article/view/2513/1631.

8. Ferreira VA, Magalhaes R. Práticas alimentares cotidianas de mulheres obesas moradoras da Favela da Rocinha (Rio de Janeiro, RJ, Brasil). Ciênc Saúde Coletiva [internet]. 2011[acesso em mar 11];16(6):2983-91. Disponível em: http://www.scielosp.org/pdf/csc/v16n6/36.pdf.

9. Petersen FB, Danilevicz, AMF. Analise qualitativa e quantitativa de atributos valorativos de empreendimentos imobiliarios em Porto Alegre. Rev Gestão Industrial, Ponta Grossa. 2006;2(4):63-74.

10. World Health Organization. Report of a WHO expert committee: the use and interpretation of antrhropometry. Geneva: World Health Organization; 1995.

11. Mendonça C. Práticas alimentares e de atividade física de mulheres obesas atendidas em unidades de saúde pública do município de Niterói: trajetórias e narrativas [tese]. Rio de Janeiro: Fundação Oswaldo Cruz, Escola Nacional de Saúde Pública; 2005.

12. Motta DG, Peres MTM, Calçada ML, Vieira CM, Tasca APW, Passarelli C. Consumo alimentar de famílias de baixa renda do município de Piracicaba/SP. Saúde em Revista. 2004;6(13):63-70.

13. Brasil. Ministério da Saúde. Guia alimentar para a população brasileira: promovendo a alimentação saudável. Brasília: Ministério da Saúde; 2006. p.22.

14. World Health Organization. Global strategy on diet, physical activity and health. FiftySeventh World Health Assembly, WHA 57.17; 2004; Geneva. Geneva; 2004.

15. Cunha IC, Peixoto MRG, Jardim PCBV, Alexandre VP. Fatores associados à prática de atividade física na população adulta de Goiânia: monitoramento por meio de entrevistas 


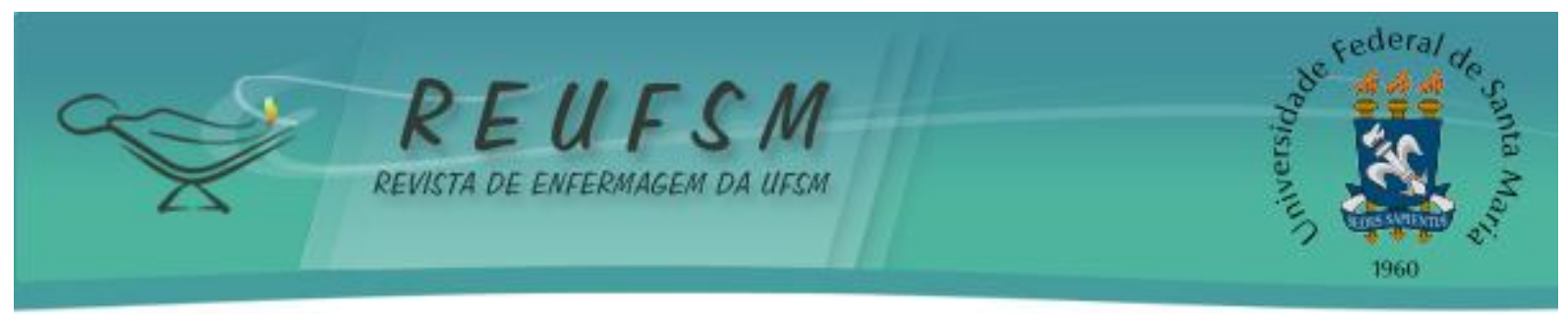

telefônicas. Rev Bras Epidemiol [internet]. 2008 [acesso em abr 11];11(3):495-504. Disponível em: http://www.scielo.br/pdf/rbepid/v11n3/15.pdf.

16. Martins TG, Assis MAA, Nahas MV, Gauche H, Moura EC. Inatividade física no lazer de adultos e fatores associados. Rev Saúde Pública [internet]. 2009 [acesso em abr 11];43(5):814-24. Disponível em: http://www.scielosp.org/pdf/rsp/v43n5/553.pdf.

17. Fett CA, Fett WCR, Marchini JS, Ribeiro RPP. Estilo de vida e fatores de risco associados ao aumento da gordura corporal de mulheres. Ciênc Saúde Coletiva [internet]. 2010 [acesso em fev11];15(1):131-40. Disponível em: http://www.scielo.br/pdf/csc/v15n1/a19v15n1.pdf.

18. Araújo, TM, Almeida, MMG, Santana, CC Araújo EM, Pinho PS. Transtornos mentais comuns em mulheres: estudo comparativo entre donas-de-casa e trabalhadoras. Rev Enferm UERJ [internet]. 2006 [acesso em fev 11];14(2):260-9. Disponível em: http://www.facenf.uerj.br/v14n2/v14n2a17.pdf.

19. Marins BR, Araujo IS, Jacob SC. A propaganda de alimentos: orientação, ou apenas estímulo ao consumo? Ciênc Saúde Coletiva [internet]. 2011 [acesso em abr 11];16(9):387382. Disponível em: http://www.scielosp.org/pdf/csc/v16n9/a23v16n9.pdf.

20. Pereira RA, Andrade RG, Sichieri R. Mudanças no consumo alimentar de mulheres do Município do Rio de Janeiro, Brasil, 1995-2005. Cad Saúde Pública [internet]. 2009 [acesso em jan 11];25(11):2419-32.. Disponível em: http://www.scielo.br/pdf/csp/v25n11/12.pdf.

21. Organização Mundial da Saúde. Necessidade de energia e proteína. Genebra: Roca; 1998.

22. Anjos LA. Obesidade e saúde pública. Rio de Janeiro: Fiocruz; 2006. p. 36.

23. SA NNB, Moura EC. Excesso de peso: determinantes sociodemográficos e comportamentais em adultos, Brasil, 2008. Cad Saúde Pública [internet]. 2011[acesso em abr 11];27(7):1380-92. Disponível em: http://www.scielo.br/pdf/csp/v27n7/13.pdf.

24. Pinho CPS, Diniz AS, Arruda IKG, Lira PIC, Sequeira LAS, Gonçalves FCLSP, et al. Excesso de peso em adultos do Estado de Pernambuco, Brasil: magnitude e fatores associados. Cad Saúde Pública, Rio de Janeiro. 2011;27(12):2340-50.

Data de recebimento: $12 / 01 / 2012$

Data de aceite: 08/05/2012

Contato com autor responsável: Nayara Mendes Cruz

Endereço: Rua Henrique Rocha, 398, Juazeiro-Bahia.

E-mail: nayaramendescruz@hotmail.com 